\title{
Does energy of type IIP supernovae depends on stellar mass?
}

\author{
N. N. Chugai ${ }^{1}$, and V. P. Utrobin ${ }^{2}$
}

\begin{abstract}
We determine the oxygen density in the central zone of nine type IIP supernovae (SN IIP) at the nebular stage using oxygen doublet [O I] 6300, $6364 \AA$. Combined with two available estimates these data indicate that oxygen densities on day 300 are distributed in rather narrow range $(2.3 \pm 1) \times 10^{9} \mathrm{~cm}^{-3}$. The result does not depend on the distance, extinction, or model assumptions. We demonstrate that the found density distribution suggests that the explosion energy of SN IIP increases with the stellar mass.
\end{abstract}

\section{Introduction}

Type IIP supernovae are caused by an explosion related with the core collapse of massive stars. The theory of stellar evolution predicts that SN IIP progenitors reside in the mass $(M)$ range of $9-25 M_{\odot}$ (Heger et al. 2003); the bounds may be in error by $20 \%$. The ejected mass $\left(M_{e}\right)$ is lower by the neutron star and the mass lost by the stellar wind. How do SN IIP explode - the question that still remains unresolved. Two major mechanism are discussed in this context: neutrino deposition (Colgate \& White 1966) and magnetorotational explosion (Bisnovatyi-Kogan 1971). A third interesting scenario suggests the rotational fragmentation of the protoneutron star followed by an explosion of neutron mini-star of $0.1 M_{\odot}$ (Imshennik 1992)

Of particular interest for the observational verification of the explosion mechanisms could be the relation between the explosion energy and progenitor mass. Common sense arguments suggests that the explosion energy should increase with the star binding energy. The latter increases with the progenitor mass (Woosley et al. 2002), so one expects in this case that the explosion energy (i.e., kinetic energy at infinity) should rise with a progenitor mass. However, the analyses of the neutrino mechanism in the framework of the 2D-hydrodynamics with a simplified neutrino transfer implies that the explosion energy, on the contrary, should decrease with the rising stellar mass at least in the progenitor mass range of $15-25 M_{\odot}$ (Fryer

\footnotetext{
${ }^{1}$ Institute of Astronomy, RAS, Pyatnitskaya 48, 109017 Moscow, Russia; nchugai@inasan.ru

${ }^{2}$ Institute of Theoretical and Experimental Physics, 117218 Moscow, Russia; utrobin@itep.ru
} 
1999). Recent numerical experiments using one-dimensional hydrodynamics with analytical description of the neutrino luminosity predict the non-monotonic $E(M)$ relation in the mass range of $10-28 M_{\odot}$ with the energy variation in the range of $(0.5-2) \times 10^{51} \mathrm{erg}$ (Ugliano et al. 2012). As to the magnetorotational mechanism, Moiseenko \& Bisnovatyi-Kogan (2012) show that the explosion energy monotonically increases with the core mass, if one admits the constant ratio of the rotational-to-gravitational energy.

A phenomenological relation between the energy and mass can be infered directly from the hydrodynamic modelling of a large sample of SN IIP. This sort of the study for the eight SN IIP indicates a correlation between the energy and progenitor mass (Utrobin \& Chugai 2013). The problem is that the explored sample comprises only progenitors with masses $>15 M_{\odot}$; whether this stems from the observational selection or the mass overestimation, remains an open issue. A different conclusion is made by Nadyozhin (2003) using estimates based on analytical relations between the observables and the supernova parameters: a sample of 14 SN IIP does not show any correlation between the explosion energy and the mass (but see Hamuy (2003)). At present therefore the issue, whether the energy of SN IIP depends on the progenitor mass, remains unclear from both observational and theoretical point of view.

In the present paper we study the issue of the energy-mass relation for SN IIP using model independent arguments. For the freely expanding supernova envelope the density in the central zone depends on the ejecta mass and energy as $\rho \propto M_{e} /(v t)^{3} \propto t^{-3} M_{e}^{5 / 2} E^{-3 / 2}$. This relation $\rho\left(E, M_{e}\right)$ implies that the presence or absence of the correlation between $E$ and $M_{e}$ could be checked by means of the density measurement at some fixed stage.

There exists a simple and efficient method for the density determination in the SN IIP enevelope at the nebular stage using [O I] 6300, $6364 \AA$ doublet. The red-to-blue flux ratio in the optically thin case is $\mathrm{R} / \mathrm{B}=1 / 3$. However, in the inner zone of SN IIP, where most of the synthesised oxygen resides, the optical depth in the [O I] $6300 \AA$ line may be large. In this case the doublet ratio $\mathrm{R} / \mathrm{B}$ can be larger than $1 / 3$. This effect was observed originally in SN 1987A and was used to estimate the density and filling factor of oxygen in this supernova (Spyromilio \& Pinto 1991; Chugai 1988).

It is noteworthy, that the doublet ratio value can be hampered by the Thomson scattering which gives rise to the red wing of [O I] $6300 \AA$ line and thus the increase of $\mathrm{R} / \mathrm{B}$ ratio at the early nebular stage $(t<200 \mathrm{~d})$ up to $\mathrm{R} / \mathrm{B}>1$ (Chugai 1992). To determine the oxygen density therefore one should use late time nebular spectra. On the other hand, at the very late epoch the doublet ratio converges to the limit $\mathrm{R} / \mathrm{B}=1 / 3$, in which case the optical depth in the $6300 \AA$ line is impossible to recover. The favorable conditions for the oxygen density determination take place at age of 250-400 days. Surprisingly, despite the 
significance of the density diagnostics upon the bases of the [O I] 6300, $6364 \AA$ doublet was recognized long ago, until now apart from SN 1987A this method has been applied only for SN 1988A and SN 1988H (Spyromilio \& Pinto 1991).

In this paper we wish to measure oxygen density for a sample of SN IIP with the nebular spectra of good quality using [O I] 6300, $6364 \AA$. As a result, we hope to recover distribution function of oxygen density $p(<n)$ for this category of supernovae. The analysis of this distribution in terms of a supernova energy and mass hopefully will permit us to draw the conclusion on the energy-mass relation and to answer the question posed by the paper title. We start with the conditions in the oxygen line-emitting zone and the method of oxygen density measurement. We find then the oxygen density for a sample of SN IIP and finaly present results for the analysis of the density distribution function.

\section{Oxygen density measurement}

The line-emitting oxygen in SN IIP undoubtedly is a product of thermonuclear helium burning in the progenitor core. Prior the supernova explosion this oxygen forms the oxygen shell on top of the iron core with the $\sim 0.2 M_{\odot}$ of silicon in between (Woosley et al. 2002). Pre-SN models predict that the oxygen mass fraction in the oxygen material is $\approx 0.8$ (Eastman et al. 1994). The additional constituents are Si, Mg, Ne, and C, with Si being dominant in the inner and $\mathrm{C}$ in the outer layers. The explosion brings about the macroscopic mixing of the oxygen material with the outer helium and hydrogen matter mediated by Rayleigh-Taylor instability (Müller et al. 1991). As a result, the oxygen turns out to be distributed in condensations imbedded in the helium and hydrogen material. Similar oxygen condensations are seen, e.g., in the Cas A supernova remnant although with higher velocities compared to SN IIP because the Cas A supernova was SN IIb (Krause et al. 2008) lacking hydrogen envelope. The [O I] doublet in SN 1987A indicates that the filling factor of oxygen condensations is $f \approx 0.1$ (Spyromilio \& Pinto 1991; Chugai 1988). Similar estimate is obtained from the flux fluctuations in the doublet profile (Chugai 1994).

The $[\mathrm{OI}] 6300,6364 \AA$ forbidden lines are emitted by transitions between the upper ${ }^{1} \mathrm{D}_{2}$ level and two lower levels, ${ }^{3} \mathrm{P}_{2}$ (ground level) and ${ }^{3} \mathrm{P}_{1}$. Generally, comparable rates of radiation and collisional transititions suggest that the population of three lower levels ${ }^{3} \mathrm{P}_{0,1,2}$ should be close to the Boltzmann one. However, given the significance of our diagnostics one needs to be sure that the assumption of the Boltzmann distribution for lower levels is justified.

We first consider the ionization and thermal balance. The ionization and heating of 
oxygen at the nebular stage is maintained by gamma-rays from the radioactive decay ${ }^{56} \mathrm{Co}$ $-{ }^{56} \mathrm{Fe}$. The fraction of the deposited energy of gamma-rays spent on the heating $(\eta)$ is taken according to Kozma \& Fransson (1992), while the energy spent on the ionization and excitation is assumed to be similar, i.e., 0.5(1- $\eta)$. The oxygen recombination coefficient is taken according to Shull \& van Steenberg (1982). The model parameters are the expansion velocity at the outer boundary of the oxygen line-emitting zone $(v)$, the amount of ${ }^{56} \mathrm{Ni}$ $\left(M_{\mathrm{ni}}\right)$, and the density. Here we adopt the density to be equal to the oxygen density. The ${ }^{56} \mathrm{Ni}$ is assumed to be distributed in the oxygen zone. The major coolant are the oxygen emission and MgII $2800 \AA$ doublet emission; the later contributes less than $10 \%$. Note that the component of the oxygen material with the carbon being dominant among minor species, cools efficiently by CO emission and does not contribute to [O I] doublet emission (Liu \& Dalgarno 1995). The observed oxygen doublet emission originates primarily from the oxygen with $\mathrm{Ne}, \mathrm{Mg}$, and Si being dominant admixtures where Mg II $2800 \AA$ is the strongest emission next to the oxygen doublet.

Our model takes into account a specific property of the central zone of SN IIP which is revealed through the failure of the model with a homogeneous mixing of ${ }^{56} \mathrm{Ni}$ to reproduce the initial $(t<300 \mathrm{~d})$ rise of the $[\mathrm{O}$ I] flux in SN 1987A (e.g. Kozma \& Fransson 1998). To account this rise one needs to admit that the ${ }^{56} \mathrm{Ni}$ is separated from the oxygen clumps by the absorbing layer devoid of the oxygen (Chugai et al. 2005). The average optical depth for gamma-quanta of this absorbing layer $\tau_{\gamma}$ we express as some fraction $\psi$ of the total optical depth of the oxygen-emitting zone $\tau_{\gamma}=\psi \tau_{\gamma, 0}$. The $\psi$ value can be found by comparing a model and observed luminosity of [O I] doublet.

The observed luminosity of the $[\mathrm{O}$ I] doublet in case of SN $1987 \mathrm{~A}$ on day 300 is $2 \times$ $10^{39} \mathrm{erg} \mathrm{s}^{-1}$ (Menzies 1991; Hanuschik 1991). Adopting $n=2 \times 10^{9} \mathrm{~cm}^{-3}$ on day 300 (Spvromilio \& Pinto 1991),$M_{\mathrm{ni}}=0.075 M_{\odot}$ (Suntzeff \& Bouchet 1991; Whitelock et al. 1991) and $v=1750 \mathrm{~km} \mathrm{~s}^{-1}$ (Chugai 1992) we find that the observed luminosity of the [O I] doublet implies $\psi=0.27$. In that case the temperature is $T=4110 \mathrm{~K}$ and ionization $x=0.018$. Below we use $\psi=0.27$ for other SN IIP as well. In the case of SN 2004et, the high luminosity SN IIP (Sahu et al. 2006), adopting $M_{\mathrm{ni}}=0.07 M_{\odot}$ and $v=1900 \mathrm{~cm} \mathrm{~s}^{-1}$ (Utrobin \& Chugai 2009) and the oxygen number density concentration $n=2 \times 10^{9} \mathrm{~cm}^{-3}$ on day 300 we find $T=3880 \mathrm{~K}$ and $x=0.016$. In the oposite case of the low luminosity SN 1997D (Turatto et al. 1998) with the parameters $M_{\mathrm{ni}}=0.005 M_{\odot}$ and $v=600 \mathrm{~cm} \mathrm{~s}^{-1}$ one gets $T=4260 \mathrm{~K}$ and $x=0.02$ assuming $n=2 \times 10^{9} \mathrm{~cm}^{-3}$ on day 300 . Note that the temperature and ionization fraction are not sensitive to the density value. These results for three different SN IIP show that the oxygen at the considered epoch is essentially neutral and the temperature is in the range of $3800-4300 \mathrm{~K}$. 
Populations of O I lower levels are found by solving rate equations taking into account four relevant levels ${ }^{3} \mathrm{P}_{0,1,2}$ and ${ }^{1} \mathrm{D}_{2}$; transition probabilities are from Aller (1984). For the temperature range $3800-4300 \mathrm{~K}$ and ionization $0.01-0.05$ the found population ratio $n_{2} / n_{1}$ of ${ }^{3} \mathrm{P}_{1}$ and ${ }^{3} \mathrm{P}_{2}$ levels obey Boltzmann law with the accuracy of $1 \%$. The upper ${ }^{1} \mathrm{D}_{2}$ level can be underpopulated by $10 \%$ compared to Boltzmann; this however does not affect the doublet $\mathrm{R} / \mathrm{B}$ ratio.

Given small deviation of the $n_{2} / n_{1}$ ratio from the Boltzmann distribution and weak sensitivity of this ratio to the temperature in the relevant range of values we adopt the Boltzmann law and $T=4000 \mathrm{~K}$ for each case below. With levels ${ }^{3} \mathrm{P}_{2},{ }^{3} \mathrm{P}_{1}$, and ${ }^{1} \mathrm{D}_{2}$ numbered 1,2 and 3 , the ratio of intensities $\left(I_{\lambda}\right)$ becomes

$$
\mathrm{R} / \mathrm{B}=\exp \left(E_{12} / k T\right)\left(\frac{\lambda_{13}}{\lambda_{23}}\right)^{5} \frac{\left[1-\exp \left(-\tau_{23}\right)\right]}{\left[1-\exp \left(-\tau_{13}\right)\right]},
$$

where $\tau_{13}$ and $\tau_{23}$ are the optical depth of 6300 and $6364 \AA$ lines respectively. The line optical depth of the homologously expanding envelope in the Sobolev approximation is determined by the local oxygen number density $\tau \propto n t$. This means that the equation (1) permits us to find the doublet ratio $\mathrm{R} / \mathrm{B}$ given the number density and, on the reverse, to determine the number density given the $\mathrm{R} / \mathrm{B}$ ratio.

The measured doublet ratio and the inferred oxygen number density for nine SN IIP are listed in Table 1 along with the spectra epochs (column 2). Note, the number density are recalculated for the epoch $300 \mathrm{~d}$ using the relation $n \propto t^{-3}$. The doublet components are partially overlapped in all but two supernovae (SN 1997D and SN 2009N), so we use the decomposition procedure with the template line profile $f \propto \exp \left(-|x|^{a}\right)$, where $x$ is the wavelength displacement relative to the line center in units of the line width $\left(\Delta \lambda / \Delta \lambda_{D}\right)$; note, the line center may be not at the rest wavelength. The power index $a$ in most cases is close to two. In some cases the doublet lines are slightly asymmetric which is taken into account by introducing a second component. We show in Fig. 1 examples of fits for SN 1987A and SN 2012A with one and two line components respectively.

The doublet ratio from Table 1 are plotted in Fig. 2 along with the model R/B ratio for several values of number density on day 300. The data of Table 1 and Fig. 2 show that the densities of eight SN IIP fall into the narrow range $(1-3) \times 10^{9} \mathrm{~cm}^{-3}$ and only one (SN 2005cs) has $n \approx 5 \times 10^{9} \mathrm{~cm}^{-3}$. Remarkably, the number density of oxygen $1.9 \times 10^{9} \mathrm{~cm}^{-3}$ for SN $1988 \mathrm{~A}$ and $1.8 \times 10^{9} \mathrm{~cm}^{-3}$ for SN 1988H (both type IIP) recovered by (Spyromilio \& Pinto 1991) fall into the above narrow range of oxygen densities.

Differential $(d N / d \log n)$ and cummulative $p(<n)$ density distributions with SN 1988A and SN 1988 H taken into account are shown in Fig. 3. The average density value is $2.3 \times 10^{9}$ 
$\mathrm{cm}^{-3}$ with the the standard deviation of $10^{9} \mathrm{~cm}^{-3}$. The relatively small scatter of density values for the sample of SN IIP of different luminosity, energy, and ejecta mass is rather suprising result. Note that the found density distribution does not depend on the distance, extinction, or other assumptions and should be considered a reliable fact.

\section{Analysis of distribution function}

\subsection{General consideration and toolkit}

The observation that the oxygen density on day 300 for different SN IIP lie in the narrow range already indicates monotonically rising function $E\left(M_{e}\right)$. Indeed, as mentioned the density in the central zone of the envelope depends on the energy and mass as $\rho \propto$ $M_{e}^{5 / 2} E^{-3 / 2}$. In the absence of any correlation between $E$ and $M_{e}$ the mass variation by a factor of two and energy by a factor of ten should cause the density variation by two order, in strong disagreement with the found density distribution $p(<n)$. The energy-mass relation $E \propto M_{e}^{5 / 3} \rho^{-2 / 3}$ that follows from above expression combined with the small variation of $\rho^{2 / 3}$ suggests that the energy should increase with the mass of the SN IIP envelope.

To make more detailed conclusions one needs to perform modelling the distribution function $p(<n)$ in the framework of reasonable assumptions. For our purpose the supernova density-velocity distribution can be described analytically $\rho=\rho_{0} /\left(1+x^{k}\right)$ where $x=v / v_{0}$ while $v_{0}$ and $\rho_{0}$ are determined by parameters $E$ and $M_{e}$. This function reproduces essential features of the density distribution in hydrodynamic models of SN IIP, particularly, a plateau in the central zone and a power law density drop in outer layers $\rho \propto v^{-k}$ where $k \approx 8$ (Utrobin 2007); below we use $k=8$.

One has to take into account that the oxygen density exceeds the average density in the line-emitting zone. The reason is that oxygen condensations form as a result of collision of the oxygen shell accelerated by the shock with the rarefied helium and hydrogen envelopes. The deceleration of the oxygen shell is accompanied by the Rayleigh-Taylor instability followed by the emergence of "fingers" of dense oxygen material protruding into the $\mathrm{He} / \mathrm{H}$ matter. According to 2D-simulation of SN 1987A explosion (Müller et al. 1991) the density contrast of oxygen fingers relative to the ambient material is $\sim 3 \ldots 10$. A similar number can be obtained from the following arguments. The main stage of the Rayleigh-Taylor instability coincides with the maxumum deceleration of the oxygen shell. For this to be the case the forward shock should sweep up the mass exceeding the mass of the oxygen shell $M_{s w}=\mu M_{\mathrm{O}}$, where presumably $\mu \sim 2$. In case of SN 1987A the filling factor of the oxygen condensation is $f \approx 0.1$ (Chugai 1988; Spvromilio \& Pinto 1991), which implies that the oxygen density 
contrast relative to the ambient material in the mixing zone is $(1-f) / f \mu \sim 5$, consistent with $2 \mathrm{D}$ simulations. This value corresponds to the contrast relative to the average density $\chi=1 / f(1+\mu) \sim 3$.

Another estimate of this value can be made via the comparison of the average density in the inner zone of a normal SN IIP, e.g., SN 1999em calculated by one-dimensional hydrodynamic model and the oxygen density derived from the [O I] doublet profile. Parameters of SN $1999 \mathrm{em}, M_{e}=19 M_{\odot}$ and $E=1.3 \times 10^{51} \mathrm{erg}$ (Utrobin 2007), with above analytical density distribution result in the average density in the central zone $\rho=2.3 \times 10^{-14} \mathrm{~g} \mathrm{~cm}^{-3}$ on day 300. At the same time, [O I] doublet ratio gives the oxygen density $\rho_{c}=6.7 \times 10^{-14} \mathrm{~g}$ $\mathrm{cm}^{-3}$ assuming the oxygen abundance of 0.8 (Table 1 ). This suggests the density contrast for the oxygen condensation $\chi=2.9$. Summarizing all estimates we expect the density contrast $\chi \sim 3$ for the oxygen condensations in the central zone of SN IIP.

We are interested in the relation between the energy and the progenitor mass $M=$ $M_{e}+M_{n s}+M_{w}$, where $M_{n s}=1.4 M_{\odot}$ is the neutron star mass and $M_{w}$ is the mass lost through the wind over the stellar life. Evolutionary computations by (Heger 2000) with the mass loss rate of Nieuwenhuijzen \& de Jager (1990) can be approximated in the range of $10-25 M_{\odot}$ by the relation $M_{w}=0.4 \beta\left(M / 10 M_{\odot}\right)^{3}$ with $\beta=1$. We however use below $\beta=0.5$. This choice is based on the $M_{w}$ value estimated for SN 2004et with the ejecta of $24 M_{\odot}$ (Utrobin \& Chugai 2009).

The density distribution $p(<n)$ is modelled by Monte Carlo dicing of $E$ and $M$. The initial progenitor masses presumably are distributed according to the Salpeter law $d N / d M \propto$ $M^{-2.35}$ in the range $M_{1}<M<M_{2}$, where for the standard case $M_{1}=9 M_{\odot}$ and $M_{2}=$ $25 M_{\odot}$. The corresponding energy range is $(0.2-4) \times 10^{51}$ erg according to parameters of the SN IIP sample studied by the hydrodynamic modelling (Utrobin \& Chugai 2013); peculiar SN 2009kf with very high explosion energy is not included in the present sample. Below we also consider other options in addition to standard choice of parameters.

\subsection{Density distribution and $E(M)$ relation}

We present here simulations of the $p(<n)$ distribution for seven options. Parameters of first four models are given in Table 2. In the first case (model m1) there is no correlation between $E$ and $M$ : for each $M$ the energy is homogeneously distributed in the range $(0.2-4) \times 10^{51} \mathrm{erg}$. The model $\mathrm{m} 2$ (standard model) suggests the power law relation $E=E_{1}\left(M / M_{1}\right)^{q}$ with the power law index determined by the interval boundaries $q=\ln \left(E_{2} / E_{1}\right) / \ln \left(M_{2} / M_{1}\right)$. We admit a scatter of energy $E(M)$ for a given mass in the 
range $E / s-s E$ where $s=1.1$. This scatter mimics an effect of possible variations of mass loss, rotation, chemical composition and magnetic field. We find however that result is not sensitive to the $s$ value. The model $\mathrm{m} 3$ differs from $\mathrm{m} 2$ by the upper boundary of mass and energy, $M_{2}=20 M_{\odot}, E_{2}=2 \times 10^{51} \mathrm{erg}$, and by the density contrast $\chi=3$. The model $\mathrm{m} 3$ approximately corresponds to the mass distribution of progenitors according to archive images (Smartt et al. 2009). The model $\mathrm{m} 4$ differs from the model $\mathrm{m} 2$ by higher mass of lower boundary, $M_{1}=15 M_{\odot}$, which corresponds to the mass range of the SN IIP sample studied hydrodynamically (Utrobin \& Chugai 2013). We considered also the model m2s in which the luminosity selection effect for the model $\mathrm{m} 2$ is added. The latter is realised via multiplying the Salpeter mass spectrum by a factor that suppresses the contribution of low mass supernovae $M<13 M_{\odot}$, presumably underluminous SN IIP. This factor is represented by $0.5[1+\tanh (x)]$, where $x=(M-12) / 2$ and $M$ is solar unit.

Keeping in mind the recent study of the energy-mass relation in the framework of neutrino mechanism (Ugliano et al. 2012) we consider models m5 and m6 that correspond to the mass and energy parameters from this paper. Specifically, models m5 and m6 take into account that in the range of $10-28 M_{\odot}$ there are two intervals, $15-16.3 M_{\odot}$ and $23-26 M_{\odot}$, in which supernovae do not explode. In the range of $10-15 M_{\odot}$ the energy increases in the interval $(0.9-1.7) \times 10^{51} \mathrm{erg}$; in the range of $16-23 M_{\odot}$ the energy is uniformly distributed in the interval of $(0.6-1.6) \times 10^{51} \mathrm{erg}$, while in the range of $26-28 M_{\odot}$ the energy is uniformly distributed in the interval of $(0.9-1.7) \times 10^{51} \mathrm{erg}$. The discribed algorithm reproduces the energy-mass relation by (Ugliano et al. 2012) with a reasonable accuracy. The only difference between models $\mathrm{m} 5$ and $\mathrm{m} 6$ is the mass loss: the model $\mathrm{m} 5$ has the mass loss adopted by (Ugliano et al. 2012) (cf. their Fig. 3), whereas the model m6 suggests the lost mass assumed in our other models (e.g. model m2). The adopted density contrast in models $\mathrm{m} 5$ and $\mathrm{m} 6$ is $\chi=3.5$.

The calculated oxygen density distributions are shown in Fig. 4 along with the observed distribution $p(<n)$. The assumption of the absence of any correlation between $E$ and $M$ (model m1) apparently contradicts to observations. On the other hand, models $\mathrm{m} 2$ and $\mathrm{m} 3$ with the power law $E(M)$ relation and the density contrast $\chi=3$ and 3.5, respectively, agree satisfactory with the observed density distribution. We performed simulations for models $\mathrm{m} 2$ and $\mathrm{m} 3$ with the $\chi$ value randomly distributed in the range $3.5 \pm 0.5$ and $3 \pm 0.5$ respectively and did not find a noticeable difference with the case of fixed contrast values. Note that multiplication of the contrast value by some factor, $\phi \chi$, results in the shift of the model distribution function by $\log \phi$ along the $\log n$ axis. In both $\mathrm{m} 2$ and $\mathrm{m} 3$ models the energy depends on mass as $E \approx 2 \times 10^{50}\left(M / 9 M_{\odot}\right)^{2.9} \mathrm{erg}$. This suggests that the density in the central zone depends on mass as $\rho \propto M^{-1.85}$, i.e., the density is larger in low mass SN IIP. This is consistent with the fact that largest density in our smaple shows SN 2005cs with 
the progenitor mass of $9-10 M_{\odot}$ (Maund et al. 2005; Li et al. 2006). The model m2s with the selection effect which reduces the contribution of low mass supernovae, $M<13 M_{\odot}$ shifts the model $\mathrm{m} 2$ towards the lower density by 0.13 dex, i.e., by a factor of 1.35 . If an observed distribution $p(<n)$ is affected by the similar selection, the original distribution can be recovered by the shift of the observed distribution towards larger density by 0.13 dex. The model $\mathrm{m} 4$ with mass and energy ranges of the hydrodynamical models for the sample of seven SN IIP shows wide range of oxygen densities incompartible with the observed distribution. Density distributions in the models $\mathrm{m} 5$ and m6 based on the energy-mass dependence from (Ugliano et al. 2012) differ significantly from the observed distribution especially in the range of small densities.

We now compare the energy-mass relation predicted by the distribution $p(<n)$ and parameters of hydrodynamic modelling. The relation $E(M)$ in the standard model $\mathrm{m} 2$ is shown on the plot $\log E-\log M$ along with parameters of hydrodynamic models (Utrobin \& Chugai 2013). We included also our parameters of SN 2012A $\left(M=15.1 M_{\odot}, E=5.2 \times 10^{50} \mathrm{erg}\right)$. The progenitor mass is derived from ejecta mass $M_{e}$ combined with neutron star and lost mass calculated according expression given in Sec. 3.1. The power law dependence $E \propto M^{2.9}$ is qualitatively consistent with the best fit relation $E \propto M^{3.8}$ for the hydrodynamic parameters of SN IIP sample. Yet the difference between values of $E$ and $M$ of two sorts of relations is apparent. If the difference is entirely due to the mass, then the mass values of the SN sample turns out to be shifted towards higher masses by a factor of $\approx 1.3$.

\section{Discussion and conclusions}

The goal of the paper was to determine the oxygen density for a sample of SN IIP using the [O I] doublet in nebular spectra and then to study the relation between energy and progenitor mass upon the bases of the recovered density distribution. It was found unexpectedly that the range of oxygen number density on day 300 is very narrow $(2.3 \pm 1) \times 10^{9}$ $\mathrm{cm}^{-3}$. Remarkably, this result does not depend on distance, extinction, or any assumptions. The modelling for the found density distribution implies that the explosion energy of SN IIP increases with the progenitor mass. This result reflects the important property of an explosion mechanism of SN IIP that should be used to constrain explosion models. It should be emphasised that we do not include into the family of SN IIP events similar to SN 1994W (Sollerman et al. 1998; Chugai et al. 2004) and SN 2009kn (Kankare et al. 2012) which mimic SN IIP by their light curves but essentially differ by their spectra.

The power index of the $E(M)$ relation found from the distribution $p(<n)$ is close to the tangent of the scattering plot of hydrodynamic parameters of SN IIP on the $\log E-\log M$ 
plane. The oxygen density distribution thus confirms the conclusion about the increase of the explosion energy with the mass that is indicated by parameters of the hydrodynamic models (Utrobin \& Chugai 2013). On the other hand, we find that the $E(M)$ relations obtained from the hydrodynamic parameters and from the distribution $p(<n)$ differ by values $E$ or/and $M$. Interestingly, in this respect, that the observed distribution $p(<n)$ is not consistent with the model in which lower boundary is $15 M_{\odot}$, i.e. equals the lower limit of the sample of SN IIP studied hydrodynamically. One of the reason of this inconsistency could be that masses derived from hydrodynamic models are overestimated. Independent argument in favor of this possibility stems from the fact that progenitor masses of hydrodynamic models lie in the range $>15 M_{\odot}$, notably larger than the lower boundary $\left(\approx 9 M_{\odot}\right)$ which theoretically are associated with SN IIP (Heger et al. 2003). This disparity is strengthend by the fact that lower boundary of progenitor masses $\left(\approx 8 M_{\odot}\right)$ extracted from the archival images (Smartt et al. 2009) is close to the theoretical lower boundary.

The conclusion that the explosion energy of SN IIP grows with the progenitor mass can serve a good observational test of the explosion theory for this category of supernovae. The current state of the theory however does not permit us to use this test with all its power. The energy-mass relation in the framework of neutrino mechanism recovered by Ugliano et al. (2012) using one-dimensional model predicts the distribution $p(<n)$ strongly unlike the observed one. Yet existing uncertainties of the neutrino mechanism do not rule out the monotonic increase of the explosion energy in the mass range $10<M<M_{u p}$, where $M_{u p}$ is poorely known value and probably lying in the range of $20 \ldots 25 M_{\odot}$ (T. Janka, private communication). In the mechanism of the mini-neutron star the energy release is invariant, $\approx 10^{51} \mathrm{erg}$ (Imshennik 1992). Since the binding energy grows with the mass this mechanism predicts decrease of the explosion energy with the mass and therefore cannot be universal for SN IIP. Currently, only magneto-rotational explosion admits the energy increase with

the mass (Moiseenko \& Bisnovatyi-Kogan 2012) which makes it an appropriate mechanism for the SNe IIP.

We are gratefull to Lina Tomasella for sending us spectra of SN 2012A.

\section{REFERENCES}

Aller L. H. 1984, Physics of thermal gaseous nebulae (Reidel: Dordrech)

Bisnovatyi-Kogan G. S. 1971, Sov. Astron., 14, 652 
Chugai N. N., Fabrika S. N., Sholukhova O. N., Goranskij V. P., Abolmasov P. K., \& Vlasyuk V. V. 2005, Astron. Lett., 31, 792

Chugai N. N. et al. 2004, MNRAS, 352, 1213

Chugai N. 1994, ApJ, 428, L17

Chugai N. 1992, Astron. Lett.,18, 239

Chugai N., Astron. Tsirkular 1988, 1525, 15

Clocchiatti A. et al. 1996, AJ, 111, 1286

Colgate S. A., White R. H. 1966, ApJ, 143, 626

Eastman R. G., Woosley S. E., Weaver T. A., \& Pinto Ph. A. 1994, ApJ, 430, 300

Fryer C. L. 1999, ApJ, 522, 413

Hamuy M. 2003, ApJ, 582, 905

Hanuschik R. W. 1991, ESO Workshop on SN 1987A and other supernovae, Ed.: I. J. Danziger and K. Kjär (ESO: Garchiong), 237

Heger, A., Fryer C. L., Woosley S. E., Langer, N., \& Hartmann D. H. 2003, ApJ, 591, 288

Heger A., Langer N., \& Woosley S. E. 2000, ApJ, 528, 368

Imshennik V. S. 1992, Sov. Astron. Lett., 18, 194

Kankare E. et al. 2012, MNRAS, 424, 855

Kozma C. \& Fransson C. 1992, ApJ, 390, 602

Kozma C. \& Fransson C. 1998, ApJ, 497, 431

Krause et al. 2008, Science, 320, 1195

Leonard D. C et al. 2002, PASP, 114, 35

Li W. et al. 2006, Apj, 641, 1060

Liu W. \& Dalgarno A. 1995, ApJ, 454, 472

Maund J. R., Smartt S. J., \& Danziger I. J. 2005, MNRAS, 364, 33 
Menzies J. W. 1991, ESO Workshop on SN 1987A and other supernovae, Ed.: I. J. Danziger and K. Kjär (ESO: Garchiong), 209

Meikle W. P. S. et al. 2011, ApJ, 732, 109

Moiseenko S. G., Bisnovatyi-Kogan G. S. 2012, IAUS, 279, 357

Müller E., Fryxell B., \& Arnett D. 1991, A\&A, 251, 505

Nadyozhin D. K. 2003, MNRAS, 346, 97

Nieuwenhuijzen H. and de Jager C. 1990, A\&A, 231, 134

Pastorello A. et al. 2009, MNRAS, 394, 2266

Phillips M. 1990, AJ, 99, 1133

Sahu D. K., Anupama G. C., Srividya S., \& Muneer S. 2006, MNRAS, 372, 1315

Shull J. M. \& van Steenberg M. 1992, ApJSS, 49, 351

Smartt S. J., Eldridge J. J., Crockett R. M., \& Maund J. R. 2009, MNRAS, 395, 1409

Sollerman J., Cumming R., \& Lundqvist P. 1998, ApJ, 493, 933

Spyromilio J. \& Pinto Ph. 1991, ESO Workshop on SN 1987A and other supernovae. Ed.: I. J. Danziger and K. Kjär (ESO: Garchiong), 423

Suntzeff N. B. \& Bouchet P. 1991, Supernovae. Ed.: S.E. Woosley (Springer-Verlag: New York), 3

Takáts K. et al. 2014, MNRAS, 438, 368

Tomasella L. et al. 2013, MNRAS, 434, 1636

Turatto M. et al. 1998, ApJ, 498, 129

Ugliano M., Janka H.-Th., Marek A., \& Arcones A. 2012, ApJ, 757, 69

Utrobin V. \& Chugai N. 2013, A\&A, 461, 233

Utrobin V. \& Chugai N. 2009, A\&A, 506, 829

Utrobin V. 2007, A\&A, 461, 233 
Whitelock P., Catchpole R., \& Feast M. 1991, Supernovae. Ed.: S.E. Woosley. 1991, (Springer-Verlag: New York), 15

Woosley S. E., Heger A., \& Weaver T. A. 2002, Rev. Mod. Phys., 74, 1015 

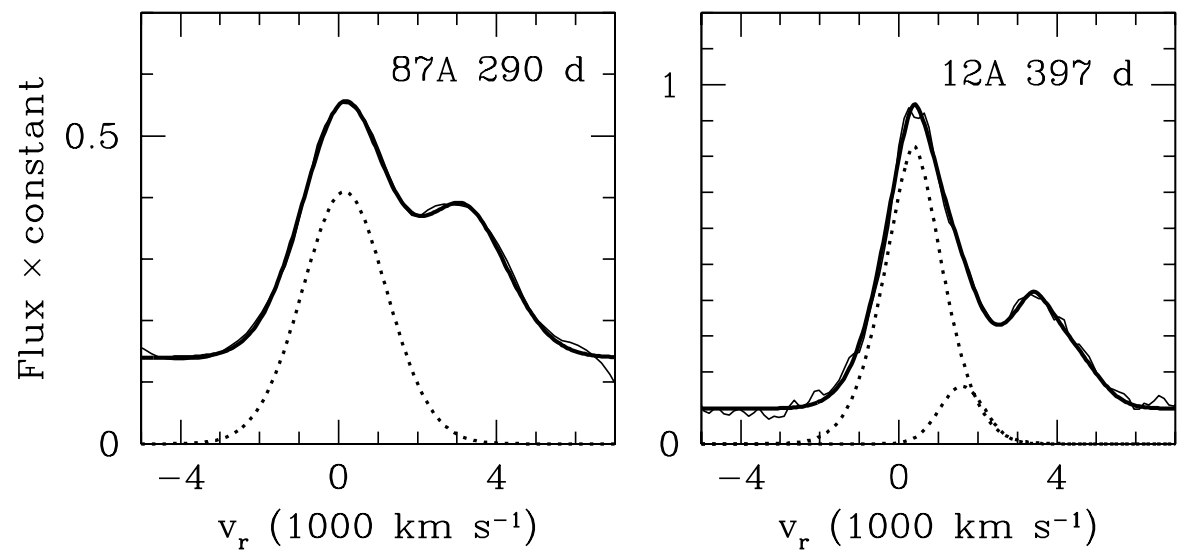

Fig. 1.- Oxygen doublet [O I] 6300, $6364 \AA$ in SN 1987A on day 290 and SN 2012A on day 397. Thin line is observations, thick line is the model with the ratio $\mathrm{R} / \mathrm{V}=0.58$. Dotted line shows the model of $6300 \AA$ line. In the case of SN 2012A the line is asymmetric and this is taken into account by the inclusion of the additional component shown by dotted line; For the dominant component $\mathrm{R} / \mathrm{V}=0.38$. 


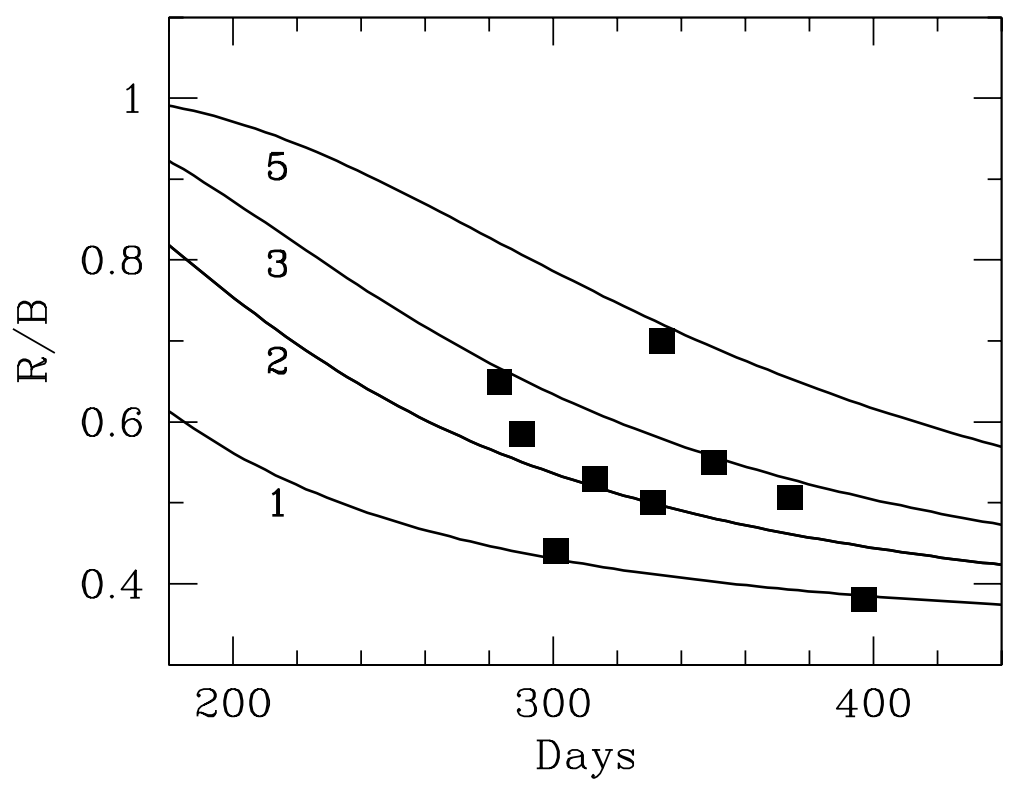

Fig. 2.- Doublet ratio R/V for the SN IIP sample at the nebular epoch (squares). Solid lines show the theoretical evolution of the ratio R/B for the oxygen number density on day 300 equals $1,2,3$, and 5 in units of $10^{9} \mathrm{~cm}^{-3}$. All the supernovae fall into this range, while 8 of 9 supernovae fall in the range $(1-3) \times 10^{9} \mathrm{~cm}^{-3}$. 

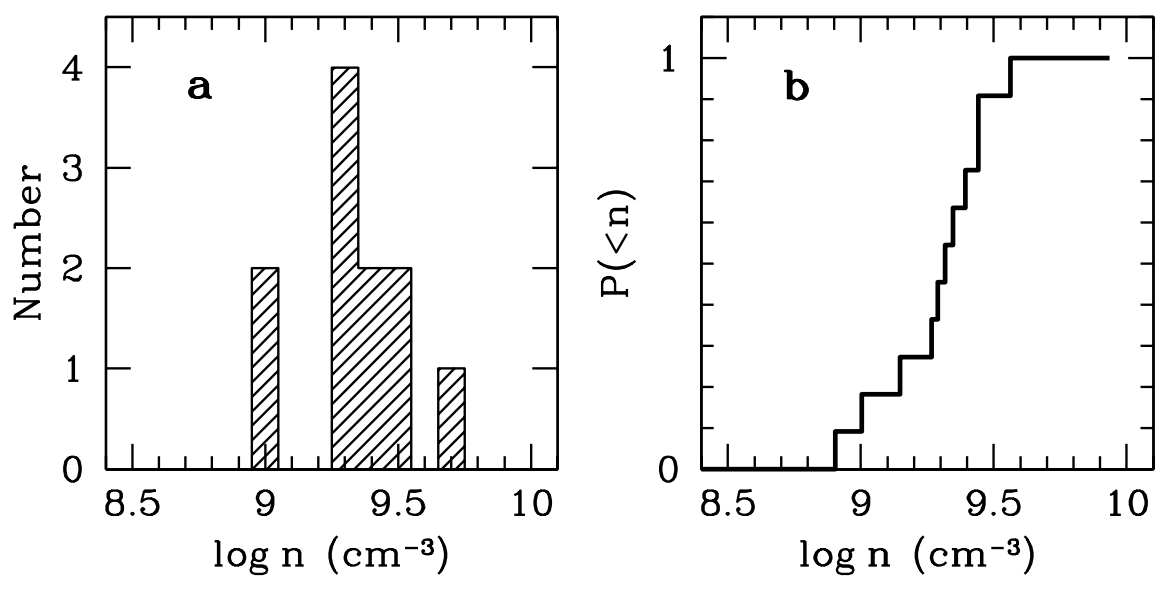

Fig. 3.- Differential (a) and cumulative (b) distribution of oxygen number density for SN IIP on day 300. The sample from Table 1 is extended by the inclusion SN 1988A and SN 1988H (Spyromilio \& Pinto 1991). 


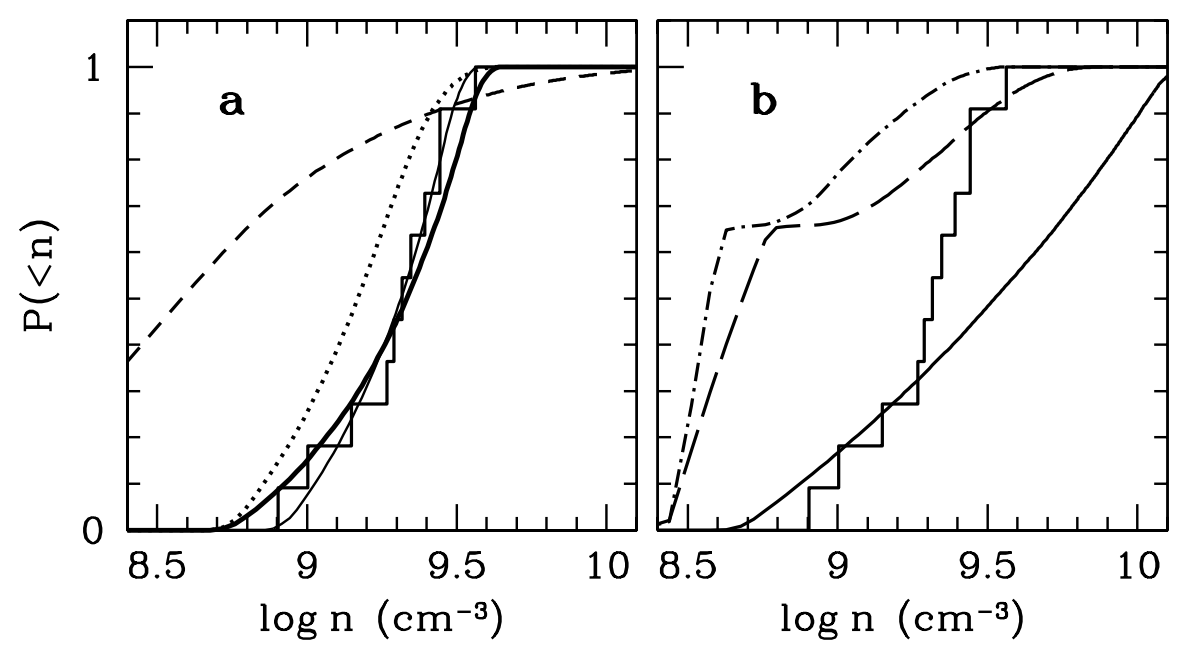

Fig. 4. - Calculated oxygen density distributions of SN IIP on day 300 compared to the observed distribution (histogram). The panel (a) shows: the model m1 (Table 2) in which no correlation between $E$ and $M$ is assumed (dashed) line; the standard model $\mathrm{m} 2$ (thick solid line); the model m3 (thin solid line), and the model m2s with the luminosity selection (dotted line). The panel (b) shows the model m4 (solid line), and two other models: m5 with strong mass loss (dash-dotted line) and the model m6 with the moderate mass loss (long dash) both based on the $E(M)$ relation reported by Ugliano et al. (2012). 


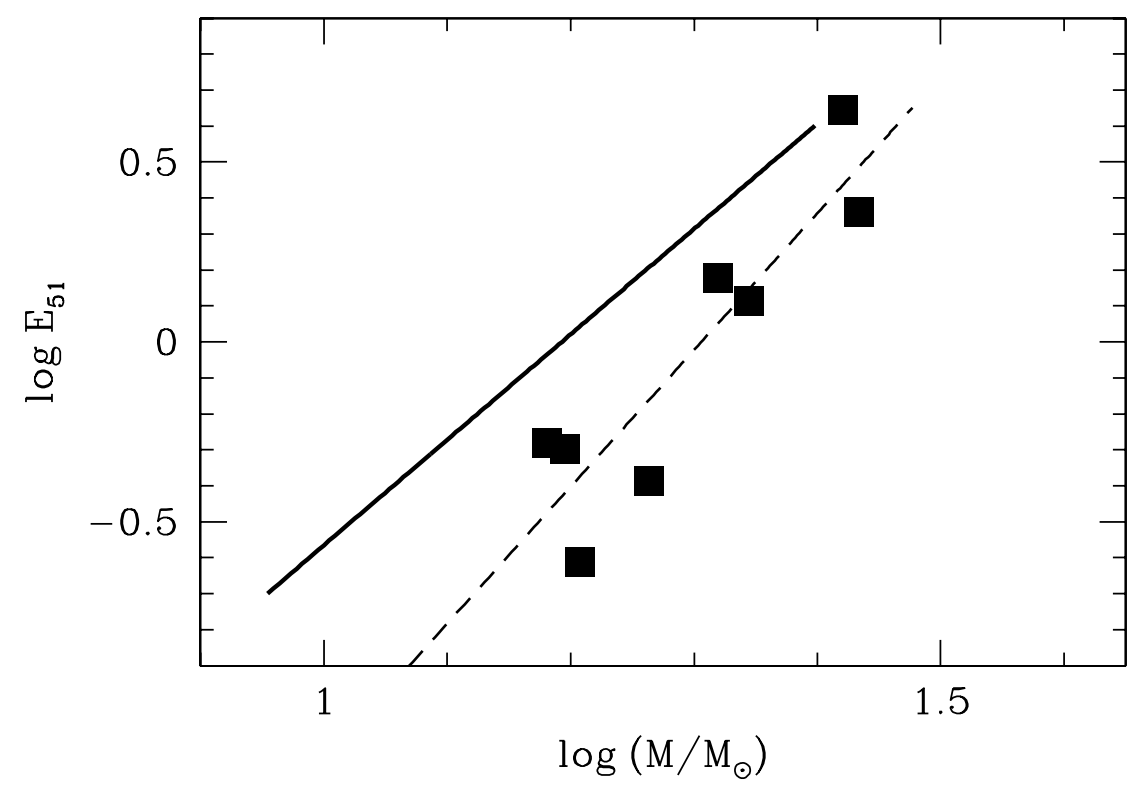

Fig. 5.- Scattering plot $E$ vs. $M$ for eight SN IIP with parameters measured by means of hydrodynamic modelling (squares). Solid line is the $E(M)$ relation of the models $\mathrm{m} 2$ and $\mathrm{m} 3$ for the distribution $p(<n)$, dashed line is linear fit for the shown SN sample. 
Table 1: Oxygen number density on day 300 for SN IIP

\begin{tabular}{ccccl}
\hline SN & $\begin{array}{c}t \\
\text { day }\end{array}$ & $\mathrm{R} / \mathrm{B}$ & $\begin{array}{c}n(300 d) \\
10^{9} \mathrm{~cm}^{-3}\end{array}$ & Spectrum \\
\hline $1987 \mathrm{~A}$ & 290 & 0.58 & 2.3 & Phillips et al (1990) \\
$1992 \mathrm{H}$ & 313 & 0.53 & 2.15 & Clocchiatti et al. (1996) \\
$1997 \mathrm{D}$ & 350 & 0.55 & 2.9 & Turatto et al. (1998) \\
$1999 \mathrm{em}$ & 331 & 0.50 & 2.0 & Leonard et al. (2002) \\
$2004 \mathrm{dj}$ & 287 & 0.65 & 2.9 & Meikle et al (2011) \\
2004et & 301 & 0.44 & 1.1 & Sahu et al. (2006) \\
$2005 \mathrm{cs}$ & 334 & 0.70 & 4.6 & Pastorello et al. (2009) \\
$2009 \mathrm{~N}$ & 374 & 0.51 & 2.65 & Takáts et al. (2013) \\
$2012 \mathrm{~A}$ & 397 & 0.38 & 0.92 & Tomasella et al. (2013) \\
\hline
\end{tabular}

Table 2: Parameters of models for oxygen density distribution

\begin{tabular}{|c|c|c|c|c|c|c|}
\hline Model & & $M_{\odot}$ & $\begin{array}{l}E_{1} \\
10^{51}\end{array}$ & $\begin{array}{l}E_{2} \\
\text { erg }\end{array}$ & $\chi$ & Comment \\
\hline$\overline{\mathrm{m} 1}$ & 9 & 25 & 0.2 & 4 & 3.5 & $E$ and $M$ do not correlate \\
\hline $\mathrm{m} 2$ & 9 & 25 & 0.2 & 4 & 3.5 & \\
\hline $\mathrm{m} 3$ & 9 & 20 & 0.2 & 2 & 3 & \\
\hline $\mathrm{m} 4$ & 15 & 20 & 0.2 & 4 & 3 & \\
\hline
\end{tabular}

